\title{
A viscoelastic constitutive model of rubber under small oscillatory load superimposed on large static deformation considering the Payne effect
}

Received: 10 June 2005 / Accepted: 7 September 2005 / Published online: 20 January 2006

(C) Springer-Verlag 2006

\begin{abstract}
The viscoelastic behavior of carbon-black-filled rubber under small oscillatory loads superimposed on large static deformation is dealt with. In this class of problems, as the strain amplitudes of the load increase, the dynamic stiffness decreases, and this phenomenon is known as the Payne effect. Besides the effects of the static deformation and the frequencies of the superimposed dynamic load, the Payne effect is considered in this study. Influence factors are introduced in this model in order to consider the influence of static predeformation, the dynamic-strain-dependent properties, and frequency-dependent properties. For simplicity, separation of the three dominant variables, frequency, prestatic deformation, and dynamic amplitude of strain, is assumed. The Kraus model is used for describing the Payne effect. Dynamic tension tests are executed to obtain the model parameters and also for the verification of the proposed model. The suggested constitutive equation shows reasonable agreement with test data.
\end{abstract}

Keywords Rubbers · Viscoelasticity $\cdot$ Constitutive equation $\cdot$ Finite prestrain $\cdot$ Payne effect

\section{Introduction}

Many rubber components are used in machinery, buildings, and large civil structures for vibration isolation. Thanks to the large damping and small stiffness of rubber, rubber isolators reduce the mechanical vibrations and transmitted forces from vibrating structures and thus prevent the fatal breakdowns of systems. On the other hand, the rubber isolators need to have a large enough degree of static stiffness because they should support the vibrating structures. Isolators generally endure small-amplitude dynamic loads on top of the large static loads caused by the self-weight of target structures. A good example is the engine mounts in automobiles and aircraft. It experiences static dead load and low-frequency forces coming from maneuvering conditions.

For large static deformations, rubber shows nonlinear elastic and nearly incompressible behavior. The nonlinear elastic behavior of rubbers can be modeled by various hyperelastic models, such as the Mooney-Rivlin form of strain energy potential. Meanwhile, rubber in vibration isolators experiences small oscillatory loads superimposed on large static deformations. Generally most of the dynamic behaviors of rubber isolators can be described by a linearized steady-state viscoelastic model. Considering the nonlinear behaviors of rubbers under large deformation, it is evident that even linearized dynamic properties depend heavily on prestrain. Morman's model is widely used to describe viscoelastic behaviors of rubbers that are under small oscillatory loads superimposed on large static deformation [1-5]. Morman's model was derived from the assumption that the time and large prestrain effects are separable. It has been observed from experiments that the separability assumption is applicable only to unfilled rubbers [1,6]; however, in filled rubbers, the relaxation function is a function of prestrain [6]. Recently Kim and Youn [7] have proposed a new viscoelastic model considering the

J.-H. Cho · S.-K. Youn $(\varangle)$

Department of Mechanical Engineering, Korea Advanced Institute of Science and Technology, 373-1, Guseong-dong,

Yuseong-gu, Daejeon 305-701, Republic of Korea

Tel.: +82-42-8693034, E-mail: skyoun@ sorak.kaist.ac.kr 
effects of prestrain in the constitutive theory of small viscoelastic motion superimposed on large static deformation in filled rubbers. This $\mathrm{K}-\mathrm{Y}$ model was derived by the linearization of simo's finite-strain viscoelastic model [8] and the introduction of the static deformation influence factor; the $\mathrm{K}-\mathrm{Y}$ model was then verified for a three-dimensional problem using finite-element analysis [9].

In general, carbon black-reinforced rubber has fairly weak frequency dependence in conjunction with pronounced amplitude dependence. If the strain amplitude increases, the storage modulus lessens and the dissipation modulus shows a more or less pronounced sigmoidal behavior, which is the well-known Payne effect [10-13]. A first model to represent and understand the Payne effect on a physical level is the so-called Kraus model $[14,15]$. The fundamental idea is that during sinusoidal deformations there is always breakage and recovery of weak physical bonds of the polymer chains. The rate of breakage is assumed to be an increasing function of the strain amplitude and the rate of recovery a decreasing function. Under stationary conditions, which are characterized by constant strain and stress amplitudes, there is a dynamic equilibrium between the rate of breakage and recovery. The storage modulus is assumed to be proportional to the total number of intact bonds and the dissipation modulus to the rate of breakage per unit of time. Some minor modifications to represent experimental data with increased accuracy have been proposed by Ulmer [15].

The purpose of this paper is to propose a viscoelastic constitutive equation of filled rubber that is under small oscillatory loads superimposed on large static deformation considering the Payne effect as well as the effects of prestrain and frequency. Influence factors are introduced to the K-Y model for this purpose. The proposed constitutive model is compared with the results from dynamic tension tests.

\section{Viscoelastic constitutive equation}

The $\mathrm{K}-\mathrm{Y}$ model [7] is a viscoelastic constitutive equation for rubber under small oscillatory load superimposed on large static deformation. However, in the model the Payne effect is not considered. The K-Y model is the linearized Simo's viscoelastic model (LSVM) with a static deformation influence factor (SDIF). LSVM was derived through linearization of Simo's model and reference transformation. The SDIF was introduced empirically to take into account the influence of prestrain on the relaxation function. It has been observed that the K-Y model agrees well with the results of dynamic compression tests [7]. In this section, we review the $\mathrm{K}-\mathrm{Y}$ model. Then the modified $\mathrm{K}-\mathrm{Y}$ model considering the Payne effect will be proposed.

\subsection{Notation}

A small deformation superimposed on a large static deformation is depicted in Fig. 1. Let $\Phi_{\xi}$ denote the configuration of the body $B$ at instant $\xi$. The $\Phi_{t^{\prime}}, \Phi_{t_{0}}$, and $\Phi_{t}$ refer respectively to the undeformed, the statically deformed, and the current configurations. The ${ }_{\xi} \mathbf{T}(\eta)$ represents a kinematical tensor $\mathbf{T}$ at time $\eta$ with respect to configuration $\Phi_{\xi}$. For convenience the following simplified notations are also used :

$$
\mathbf{T}(\eta)={ }_{t^{\prime}} \mathbf{T}(\eta),{ }_{\xi} \mathbf{T} 0={ }_{\xi} \mathbf{T}\left(t_{0}\right),{ }_{0} \mathbf{T}(\xi)={ }_{t_{0}} \mathbf{T}(\xi),{ }_{\xi} \mathbf{T}={ }_{\xi} \mathbf{T}(t) .
$$

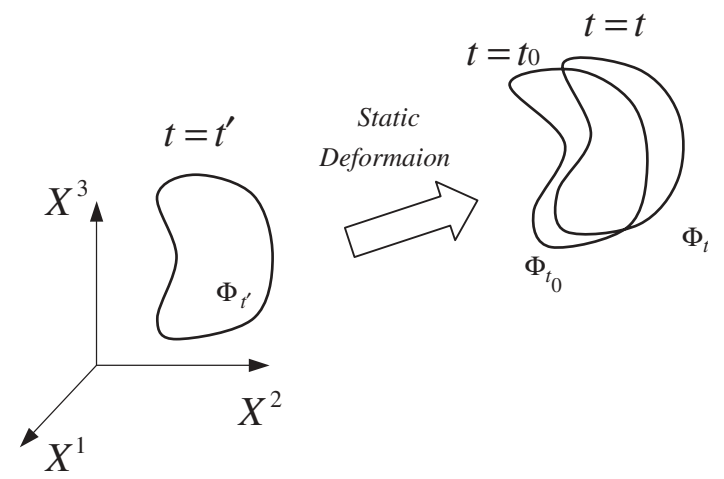

Fig. 1 Small dynamic deformation superimposed on large static deformation 
The deformation gradient tensor and the volume-preserving deformation gradient tensor are respectively denoted by

$$
\left({ }_{\xi} \mathbf{F}(t)\right)_{i j}=\left(\frac{\partial x_{i}(t)}{\partial X_{j}(\xi)}\right) \text { and }{ }_{\xi} \overline{\mathbf{F}}=J^{-1 / 3}{ }_{\xi} \mathbf{F},
$$

where $J$ is det $\left({ }_{\xi} \mathbf{F}\right)$. Left and right Cauchy-Green tensors corresponding to $\overline{\mathbf{F}}_{\xi}$ and ${ }_{\xi} \mathbf{F}$ are, respectively,

$$
\begin{gathered}
\xi \mathbf{C}={ }_{\xi} \mathbf{F}^{T}{ }_{\xi} \mathbf{F}, \quad{ }_{\xi} \overline{\mathbf{C}}={ }_{\xi} \overline{\mathbf{F}}^{T} \bar{\xi}_{\xi} \text { and } \\
\xi \mathbf{B}={ }_{\xi} \mathbf{F}_{\xi} \mathbf{F}^{T}, \quad{ }_{\xi} \overline{\mathbf{B}}={ }_{\xi} \overline{\mathbf{F}}_{\xi} \overline{\mathbf{F}}^{T},
\end{gathered}
$$

and Green Strain tensors are defined by, respectively,

$$
\xi \mathbf{E}=\frac{1}{2}\left({ }_{\xi} \mathbf{C}-\mathbf{I}\right) \quad \text { and } \quad \xi \overline{\mathbf{E}}=\frac{1}{2}(\xi \overline{\mathbf{C}}-\mathbf{I}),
$$

where $\mathbf{I}$ is the identity tensor.

\section{$2.2 \mathrm{~K}-\mathrm{Y}$ steady-state viscoelastic model}

The K-Y steady-state viscoelastic model can be derived by the linearization of Simo's finite-strain viscoelastic model and the introduction of the static deformation influence factor. Simo proposed a finitely deformable viscoelastic model [8] from the generalization of a standard linear solid. The characteristics of Simo's model are decoupled bulk and deviatoric responses over any range of deformations and a linear rate constitutive equation. Simo's nonlinear viscoelastic constitutive model is written as follows:

$$
\begin{aligned}
\mathbf{S}= & J \mathbf{C}^{-1} \frac{\partial U}{\partial J}+J^{-2 / 3} \operatorname{DEV}\left[\left(\frac{\partial \Psi}{\partial \overline{\mathbf{E}}}\right)\right] \\
& +J^{-2 / 3} \operatorname{DEV}\left[\int_{0}^{t} g(t-\xi) \frac{\partial}{\partial \xi} D E V\left(\frac{\partial \Psi}{\partial \overline{\mathbf{E}}}\right) d \xi\right],
\end{aligned}
$$

where $\operatorname{DEV}[\bullet]=(\bullet)-\frac{1}{3}[\mathbf{C}:(\bullet)] \mathbf{C}^{-1}$, and $U$ and $\Psi$ are the volumetric and deviatoric parts of the elastic free energy function. The relaxation function $g(t)$ is connected with the shear relaxation modulus of linear viscoelasticity, $G(t)$, by

$$
g(t)=\frac{G(t)}{G_{\infty}}-1
$$

where $G_{\infty}=\lim _{t \rightarrow \infty} G(t)$.

From this model static stress is given as

$$
\boldsymbol{\sigma}_{0}=P_{0} \mathbf{I}+\operatorname{dev}\left[\widehat{\boldsymbol{\sigma}}_{0}\right]
$$

where $P_{0}=\frac{\partial U}{\partial J}, \widehat{\boldsymbol{\sigma}}_{0}=\frac{1}{J} \overline{\mathbf{F}}_{0} \frac{\partial \Psi}{\partial \overline{\mathbf{E}}} \overline{\mathbf{F}}_{0}^{T}$, and $\operatorname{dev}[\bullet]=(\bullet)-\frac{1}{3}[\mathbf{I}:(\bullet)] \mathbf{I}$. We know from the above equation that the static behavior of Simo's model is exactly equal to that of hyperelastic material, for which free energy is defined as $\hat{\Psi}=U+\Psi$. The static stress caused by prestrain is determined only by the last state of static deformation, not by the deformation history. Because of the fading memory hypothesis $[16,17]$ of the viscoelastic material, the effects of the deformation history are relaxed out. Thus we can consider that viscoelastic effects depend only on superimposed small vibratory deformation.

A linearized constitutive equation that specifies the behavior of rubber under small oscillatory load superimposed on large static deformation can be derived from the assumption that the superimposed motion is 
small. Repeated applications of the chain rule to Eq. (1) and a reference configuration transformation yield the relation between the stress increment $\Delta_{0} \mathbf{S}$ and superposed strain increment $\varepsilon$ [7].

$$
\begin{aligned}
\Delta_{0} \mathbf{S}= & \left(J \frac{\partial^{2} U}{\partial J^{2}}+P\right) \varepsilon_{k k} \mathbf{I}-2 P \mathcal{\varepsilon} \\
& -\frac{2}{3}\left[\operatorname{dev}\left(\widehat{\boldsymbol{\sigma}}_{0}\right) \otimes \mathbf{I}+\mathbf{I} \otimes \operatorname{dev}\left(\widehat{\boldsymbol{\sigma}}_{0}\right)\right]: \mathcal{\varepsilon}+\mathscr{C}: \mathcal{\varepsilon}+\int_{0}^{t} g(t-\xi) \mathscr{C}: \dot{\varepsilon} \mathrm{d} \xi
\end{aligned}
$$

The superposed strain increment is defined by the displacement increments with the following equation:

$$
\varepsilon_{i j}=\frac{1}{2}\left[\frac{\partial \Delta u_{i}}{\partial x_{j}}+\frac{\partial \Delta u_{j}}{\partial x_{i}}\right] .
$$

In Eq. (3), the Truesdell elasticity tensor is given as

$$
\begin{aligned}
\mathscr{C}= & \frac{2}{3}(\widehat{\boldsymbol{\sigma}}: \mathbf{I})\left(\hat{\mathbf{I}}-\frac{1}{3} \mathbf{I} \otimes \mathbf{I}\right) \\
& +\mathscr{F}-\frac{1}{3}[(\mathscr{F}: \mathbf{I}) \otimes \mathbf{I}+\mathbf{I} \otimes(\mathscr{F}: \mathbf{I})]+\frac{1}{9}(\mathbf{I}: \mathscr{F}: \mathbf{I}) \mathbf{I} \otimes \mathbf{I},
\end{aligned}
$$

where $\mathscr{F}_{i j k \ell}=\frac{1}{J} \bar{F}_{i I} \bar{F}_{j J} \bar{F}_{k K} \bar{F}_{l L}\left(\frac{\partial^{2} \Psi}{\partial \overline{\mathbf{E}}^{2}}\right)_{I J K L}$ and $\hat{\mathbf{I}}$ is the fourth-order identity tensor.

The relation between the Cauchy stress increment, $\Delta \sigma$, and $\Delta_{0} \mathbf{S}$ is written as follows: [21]

$$
\Delta \boldsymbol{\sigma}=-\varepsilon_{k k} \sigma_{0}+\varepsilon \cdot \sigma_{0}+\sigma_{0} \cdot \varepsilon+\omega \cdot \sigma_{0}-\sigma_{0} \cdot \omega+\Delta_{0} \mathbf{S},
$$

where $\omega_{i j}=\frac{1}{2}\left(\frac{\partial \Delta u_{j}}{\partial x_{i}}-\frac{\partial \Delta u_{i}}{\partial x_{j}}\right)$.

When viscoelastic material is incompressible, we can write a modified form of the linearized Simo model:

$$
\stackrel{\operatorname{Tr}}{\Delta \sigma}=\Delta \boldsymbol{\sigma}-\varepsilon \cdot \sigma_{0}-\sigma_{0} \cdot \varepsilon-\omega \cdot \sigma_{0}+\sigma_{0} \cdot \omega=-\Delta P \mathbf{I}+\mathscr{C}: \varepsilon+\int_{0}^{t} g(t-\xi) \mathscr{C}: \dot{\varepsilon} \mathrm{d} \xi,
$$

where the pressure increment $\Delta P=-\Delta \sigma_{k k}$ is determined by boundary conditions.

Let us suppose that superimposed deformation is steady-state harmonic such as $\varepsilon=\varepsilon^{*} \mathrm{e}^{i \omega t}$. Then the complex constitutive relation is extracted from Eq. (3):

$$
\begin{aligned}
\Delta_{0} \mathbf{S}^{*}= & \left(J \frac{\partial^{2} U}{\partial J^{2}}+P\right) \varepsilon_{k k}^{*} \mathbf{I}-2 P \varepsilon^{*} \\
& -\frac{2}{3}[\operatorname{dev}(\widehat{\boldsymbol{\sigma}}) \otimes \mathbf{I}+\mathbf{I} \otimes \operatorname{dev}(\widehat{\boldsymbol{\sigma}})]: \varepsilon^{*}+\left(1+i \omega g^{*}\right) \mathscr{C}: \varepsilon^{*},
\end{aligned}
$$

where $g^{*}$ is the Fourier transform of $g(t)$, i.e., $g^{*}(\omega)=\int_{0}^{\infty} g(t) e^{-i \omega t} \mathrm{~d} t$. The relation between $g^{*}$ and complex shear modulus $G^{*}=G^{\prime}+i G^{\prime \prime}$ is as follows:

$$
\omega \mathrm{g}^{*}=\frac{G^{\prime \prime}}{G_{\infty}}+\left(1-\frac{G^{\prime}}{G_{\infty}}\right) i
$$

Thus far it has been assumed that $g(t)$, which represents the time effects, is not affected by static deformation. This separability assumption is suitable for rubbers that do not contain filler such as carbon black [6]. But for filled rubber it is known from experiments that $g(t)$ depends on the static deformation [6]. Unfilled rubber is seldom used for engineering applications. In order to describe the nonseparability nature of filled rubber, a static deformation influence factor $c^{*}\left(\mathbf{B}_{0}\right)$ is introduced into the constitutive equation and the frequency-dependent term $1+i \omega g^{*}$ is corrected as follows:

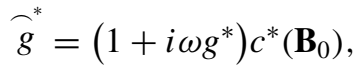

where $c^{*}\left(\mathbf{B}_{0}\right)$ is a complex valued function that depends on the static deformation. The static deformation influence factors can be depicted by the modulus and argument of $c^{*}$ :

$$
c^{*}\left(\mathbf{B}_{0}\right)=c \mathrm{e}^{i \theta} .
$$


To define $c^{*}$ in a specific form, the static deformation measure should be described by the left Cauchy-Green tensor $\mathbf{B}_{0}$. It is observed that the generalized octahedral shear strain performs well as a static deformation measure. The generalized octahedral shear strain [18] is defined as

$$
\bar{I}_{\gamma}=\frac{1}{6}\left(2 \bar{I}_{1}^{2}-6 \bar{I}_{2}\right)^{1 / 2},
$$

where $\bar{I}_{1}$ and $\bar{I}_{2}$ are first and second invariants of the right Cauchy-Green tensor $\overline{\mathbf{C}}$. The $\bar{I}_{\gamma}$ is an invariant of $\mathbf{B}_{0}$ and represents the octahedral shear strain under infinitesimal deformation. Since the value of $c^{*}$ is unity without the static deformation, the following polynomial forms can serve as the static deformation influence factors:

$$
\begin{gathered}
c_{\gamma}^{*}\left(\bar{I}_{\gamma}\right)=c_{\gamma} \mathrm{e}^{i \theta_{\gamma}}, \\
c_{\gamma}\left(\bar{I}_{\gamma}\right)=1+z_{\gamma_{1}} \bar{I}_{\gamma}+z_{\gamma_{2}} \bar{I}_{\gamma}^{2}, \\
\theta_{\gamma}\left(\bar{I}_{\gamma}\right)={ }_{\theta} z_{\gamma} \bar{I}_{\gamma},
\end{gathered}
$$

where $z_{\gamma_{1}}, z_{\gamma_{2}}$, and $\theta z_{\gamma}$ are material constants. The material constants can be easily determined by the results of a uniaxial tension test.

\subsection{Viscoelastic constitutive model considering the Payne effect}

Until now, $g(t)$, which represents the time effects, has been considered to be affected by static deformation and frequency only. But the behavior of carbon-black-filled elastomers under dynamic deformation is strongly dependent on the dynamic amplitude [10-13]. Therefore the effects on $g(t)$ due to static deformation and dynamic amplitude must be considered in order to develop a realistic viscoelastic constitutive model.

In order to describe the effects of frequency, prestrain, and dynamic amplitude, we empirically introduce three factors to the constitutive equation. In this paper the separation of each factor is assumed. The verification will be carried out by comparing the results of a tension test with those of the proposed constitutive equation. If the separation of variables is assumed, we can define $\tilde{g}^{*}$, which is the correction of $1+i \omega g^{*}$ in Eq. (4):

$$
\tilde{g}^{*}\left(\omega, \varepsilon, \mathbf{B}_{0}\right)=\tilde{g}_{\omega}^{*}(\omega) \tilde{g}_{\varepsilon}^{*}(\varepsilon) c^{*}\left(\mathbf{B}_{0}\right),
$$

where $\tilde{g}_{\varepsilon}(\varepsilon)$ is a dynamic amplitude influence factor (DAIF), $\tilde{g}_{\omega}(\omega)$ a frequency influence factor (FIF), and $c^{*}\left(\mathbf{B}_{0}\right)$ a static deformation influence factor (SDIF). In this paper, a polynomial function of log frequency is used for the type of $\tilde{g}_{\omega}(\omega)$. And Kraus model is used for $\tilde{g}_{\varepsilon}(\varepsilon)$. Finally, $c^{*}\left(\mathbf{B}_{0}\right)$ is a static deformation influence factor used in the K-Y model.

For simplicity, $\tilde{g}_{\omega}(\omega)$ is considered to be unity at reference frequency $\omega_{\text {ref }}$. The following polynomial forms can serve as FIFs:

$$
\tilde{g}_{\omega}(\omega)=\left(1+\sum_{i=1}^{n} a_{i}\left(\omega / \omega_{\mathrm{ref}}\right)^{i}\right)+i\left(\sum_{i=1}^{m} b_{i}\left(\omega / \omega_{\mathrm{ref}}\right)^{i}\right) .
$$

Now we will review the Kraus model in more detail. Kraus [14] suggested a phenomenological model to describe the dynamic strain dependence of carbon-black-filled rubbers. He assumed that aggregate-aggregate contacts (van der Waals interactions) were continuously broken and reformed under a periodic sinusoidal strain, $\gamma=\gamma_{0} \sin (\omega t)$, at fixed frequency and temperature. $\gamma_{0}$ is the magnitude of a sinusoidal strain. Therefore, in filled rubbers above the percolation threshold, the carbon black aggregates form a temporary secondary network within the rubbery polymer network. A deagglomeration rate (breakage rate) $R_{d}$ is assumed to be proportional to the number of consisting contacts $N$ and to some power $m$ of the amplitude of the strain, $\gamma_{0}^{m}$. The reagglomeration rate $R_{r}$ is assumed to be proportional to the difference between the number of possible (elastically effective) contacts at zero deformation $N_{0}$ and the number of existing contacts $N$. Furthermore, $R_{r}$ is assumed to be proportional to $\gamma_{0}^{-m}$. Then we have the following:

Deagglomeration rate: $R_{\mathrm{d}}=k_{d} \gamma_{0}^{m} N$.

Reagglomeration rate: $R_{\mathrm{r}}=k_{r} \gamma_{0}^{-m}\left(N_{0}-N\right)$, where $k_{d}$ and $k_{r}$ are the rate constants. 

obtain

Deagglomeration and reagglomeration rates are equal at the rate equilibrium, that is, $R_{d}=R_{r}$. Then we

$$
N=\frac{N_{0}}{1+\left(\gamma_{0} / \gamma_{\mathrm{c}}\right)},
$$

where $\gamma_{c}$ is a characteristic strain amplitude.

Assuming that the excess modulus of the agglomeration network is proportional to the number of currently existing contacts, one obtains

$$
G^{\prime}-G_{\infty}^{\prime} \propto N
$$

And thus,

$$
\frac{G^{\prime}-G_{\infty}^{\prime}}{G_{0}^{\prime}-G_{\infty}^{\prime}}=\frac{N}{N_{0}}=\frac{1}{1+\left(\gamma_{0} / \gamma_{c}\right)^{2 m}},
$$

where $G_{0}^{\prime}$ is the storage modulus for zero deformation and $G_{\infty}^{\prime}$ is the corresponding modulus for large deformations (plateau value at large strains). From Eq. (10), $G^{\prime}$ is written as follows:

$$
G^{\prime}\left(\gamma_{a}\right)=G_{\infty}^{\prime}+\frac{\left(G_{0}^{\prime}-G_{\infty}^{\prime}\right)}{1+\left(\gamma_{0} / \gamma_{c}\right)^{2 m}}
$$

This phenomenological formula for the strain dependency has been successfully applied to carbon-black-filled vulcanizates.

The four parameters of Eq. (11) have the following physical meanings: $G_{\infty}^{\prime}$ is the storage modulus of the pure rubber matrix increased by the hydrodynamic filler effect and specific filler-matrix interactions, $\Delta G^{\prime}\left(=G_{0}^{\prime}-G_{\infty}^{\prime}\right)$ is the excess storage modulus of the temporary secondary filler network (which will be destroyed with increasing strain amplitude), and $\gamma_{\mathrm{c}}$ is a characteristic strain amplitude, determined mainly from the ratio of the rate constants. The loss modulus maximum is at $\gamma_{\mathrm{c}}$. The $m$ exponent has been introduced by Kraus as an empirical parameter. This exponent can be related to the connectivity of the filler network and can be determined from the fractal dimension of the percolation clusters. The study of different carbon-black-filled rubber compounds yields an exponent $m$ of about 0.6. Obviously, within a certain range the amount of $m$ is nearly independent of temperature, frequency, and carbon black loading. However, $m$ depends on the embedding polymer matrix.

Formulas for $G^{\prime \prime}$ and $\tan \delta$ can be obtained in a similar manner, for example,

$$
G^{\prime \prime}(\gamma)=G_{\infty}^{\prime \prime}+\frac{2\left(G_{m}^{\prime \prime}-G_{\infty}^{\prime \prime}\right)\left(\gamma_{0} / \gamma_{\mathrm{c}}\right)^{m}}{1+\left(\gamma_{0} / \gamma_{\mathrm{c}}\right)^{2 m}} .
$$

Some minor modifications to Eq. (12) to represent experimental data with increased accuracy have been proposed by Ulmer [15]:

$$
G^{\prime \prime}(\gamma)=G_{\infty}^{\prime \prime}+\frac{2\left(G_{m}^{\prime \prime}-G_{\infty}^{\prime \prime}\right)\left(\gamma_{0} / \gamma_{c}\right)^{m}}{1+\left(\gamma_{0} / \gamma_{c}\right)^{2 m}}+\Delta G_{2}^{\prime \prime} \mathrm{e}^{-\gamma / \gamma_{2}}
$$

In this paper, (11) and (13) are used as a fitting function for describing the influence of the dynamic amplitude of strain. $G_{0}^{\prime}, G_{\infty}^{\prime}, \gamma_{c}, m, G_{m}^{\prime \prime}, G_{\infty}^{\prime \prime}, \Delta G_{2}^{\prime \prime}$, and $\gamma_{2}$ are material constants, which can be easily determined by the results of uniaxial dynamic tension tests.

\section{Material test}

\subsection{Dynamic tension test}

A dynamic tension test is carried out in order to verify the proposed constitutive model and to observe the viscoelastic behavior of the rubber. The rubber specimen recipe is shown in Table 1 . The specimen is a rectangular parallelepiped-shaped rubber element whose width, depth, and height are, respectively, 20, 20, and $160 \mathrm{~mm}$. Aluminum plates are bonded to both ends of the rubber specimen by quick-set adhesive, and each 
Table 1 Recipe of specimen (unit: phr)

\begin{tabular}{llllllllll}
\hline Rubber & ZnO & Stearic acid & 3C & RD & SUNOC 315 & Carbon black SRF & 0005C & NBS & DM \\
\hline SMR-10 & 5 & 1 & 1 & 1 & 2 & 50 & 4.4 & 1 & 0.3 \\
\hline
\end{tabular}

plate is bolted to the test machine. The tests are performed at room temperature $\left(20^{\circ} \mathrm{C}\right)$ using a servohydraulic rubber tester (Instron-5802).

To subject the specimen to small dynamic motion superimposed on finite static deformation, experiments were conducted in two steps. Finite prestrain was applied to each specimen, with 20 min of relaxation time in order to achieve the static equilibrium. Then the dynamic load was superimposed on the static deformation. The stretch values of the static tension were $1,1.05,1.10,1.15,1.2,1.25$, and 1.3. The dynamic strain amplitude values, which are 34 different points between $0.01 \%$ and $1.0 \%$ with respect to deformed specimen length, were superimposed on static deformation in the $1-100 \mathrm{~Hz}$ frequency range. Initial conditioning was applied for $12 \mathrm{~h}$ in advance before the experiment. For the initial conditioning, each test specimen was exposed to the highest strain and frequency in the test series in order to remove irreversible material structures [19]. The measurement was made after 50 cycles of dynamic loading. When the test was executed using the displacement-control mode, the dynamic displacements and the corresponding dynamic driving forces were measured as the experimental data. From these test data the dynamic modulus and stiffness were calculated considering the size of specimens.

\subsection{Calculation using constitutive equation}

\subsubsection{Constitutive equation under uniaxial deformation}

In the calculation the rubber is treated as incompressible Mooney-Rivlin material. Under this assumption the free energy function is defined as

$$
\Psi=c_{1}\left(I_{1}-3\right)+c_{2}\left(I_{2}-3\right),
$$

where $c_{1}$ and $c_{2}$ are determined by curve fitting the static axial force of the experiment. In the case of uniaxial deformation, the static stress, Finger strain tensor, incremental strain, and incremental rotation are, respectively, given as follows:

$$
\begin{gathered}
\left\{\begin{array}{l}
\sigma_{0_{11}} \\
\sigma_{0_{22}} \\
\sigma_{0_{33}}
\end{array}\right\}=\left\{\begin{array}{c}
2 c_{1}\left(\lambda^{2}-\lambda^{-1}\right)+2 c_{2}\left(\lambda-\lambda^{-2}\right) \\
0 \\
0
\end{array}\right\}, \\
\mathbf{B}_{0}=\left[\begin{array}{ccc}
\lambda^{2} & 0 & 0 \\
0 & \lambda^{-1} & 0 \\
0 & 0 & \lambda^{-1}
\end{array}\right], \\
\mathcal{E}=\left[\begin{array}{ccc}
\varepsilon_{x} & 0 & 0 \\
0 & -\frac{\varepsilon_{x}}{2} & 0 \\
0 & 0 & -\frac{\varepsilon_{x}}{2}
\end{array}\right], \\
\omega=0
\end{gathered}
$$

where $\lambda$ is stretch. The above equations and boundary conditions, $\sigma_{22}=\sigma_{33}=0$, lead to a one-dimensional form of a linearized Simo model. From Eq. (4) we obtain

$$
\Delta \sigma_{11}=\left(2 c_{1}\left(\lambda^{2}-\lambda^{-1}\right)+2 c_{2}\left(\lambda-\lambda^{-2}\right)\right) \varepsilon_{x}+\mathscr{C}_{\ell} \varepsilon_{x}+\int_{0}^{t} g(t-\xi) \mathscr{C}_{\ell} \dot{\varepsilon}_{x} \mathrm{~d} \xi
$$


where $C_{l}=2 c_{1}\left(\lambda^{2}+2 \lambda^{-1}\right)+6 c_{2} \lambda^{-2}$. When the superimposed loading is steady-state harmonic, the following complex one-dimensional constitutive model can be derived using Eq. (15) and influence factors:

$$
\Delta \boldsymbol{\sigma}_{11}^{*}=\left(2 c_{1}\left(\lambda^{2}-\lambda^{-1}\right)+2 c_{2}\left(\lambda-\lambda^{-2}\right)\right) \varepsilon_{x}^{*}+\tilde{g}^{*} \mathscr{C}_{\ell} \varepsilon_{x}^{*},
$$

where $\tilde{g}^{*}$ is defined by Eq. (8). Also $\tilde{g}^{*}$ is represented by complex shear modulus and influence factors as shown in Eqs. (6), 7), (9), (11) and (13).

\subsubsection{Determination of the parameters in constitutive equation}

To predict the behavior of a rubber specimen using a constitutive equation, we need to determine the material characteristic functions $\left(c_{1}, c_{2}, \tilde{g}^{*}\right) . c_{1}$ and $c_{2}$ are determined by curve fitting the axial force from a simple static tension test.

The constants of $\tilde{g}^{*}$ are determined by curve fitting from dynamic tension tests. At first, the constants of $\tilde{g}_{\varepsilon}(\varepsilon)$ can be derived from the results of the tension test at zero static deformation and at reference frequency. If there is no static deformation, $c^{*}\left(\mathbf{B}_{0}\right)$ is unity. Thus the constitutive equation is:

$$
\Delta \boldsymbol{\sigma}_{11}^{*}=6 \tilde{g}^{*}\left(\omega, \varepsilon, \mathbf{B}_{0}\right)\left(\mathrm{c}_{1}+\mathrm{c}_{2}\right) \varepsilon_{x}^{*}=3 G_{\infty} \tilde{g}_{\omega}(\omega) \tilde{g}_{\varepsilon}(\varepsilon) \varepsilon_{x}^{*} .
$$

Because $\tilde{g}_{\omega}^{*}(\omega)$ is set to unity at reference frequency $\omega_{\text {ref }}$, i.e., $\tilde{g}_{\omega}^{*}\left(\omega_{\text {ref }}\right)=1, \tilde{g}_{\varepsilon}(\varepsilon)$ at reference frequency is written as follows:

$$
\tilde{g}_{\varepsilon}(\varepsilon)=\frac{\left.\Delta \sigma_{11}\right|_{\omega=\omega_{\mathrm{ref}}}}{3 G_{\infty} \varepsilon_{x}^{*}}=\frac{1}{3 G_{\infty}} \frac{\left.\Delta F^{*}\right|_{\omega=\omega_{\mathrm{ref}}}}{A_{0} \varepsilon_{x}^{*}},
$$

where $\Delta F^{*}$ is the amplitude of the measured dynamic force and $A_{0}$ the cross-section area of the specimen at undeformed state. $\tilde{g}_{\varepsilon}(\varepsilon)$ is calculated at fixed reference frequency $\left(\omega_{\text {ref }}=1 \mathrm{~Hz}\right)$ with zero static deformation with changing dynamic amplitudes. The constants $\tilde{g}_{\varepsilon}(\varepsilon)$ are determined by least-square curve fitting.

The constants $\tilde{g}_{\omega}(\omega)$ can be derived from the results of a tension test at zero static deformation and at reference dynamic amplitude. From Eq. (16), $\tilde{g}_{\omega}(\omega)$ is written as follows:

$$
\begin{gathered}
\Delta \boldsymbol{\sigma}_{11}^{*}=3 G_{\infty} \tilde{g}_{\omega}(\omega) \tilde{g}_{\varepsilon}\left(\varepsilon_{\mathrm{ref}}^{*}\right) \varepsilon_{\mathrm{ref}}^{*} . \\
\tilde{g}_{\omega}(\omega)=\frac{\left.\Delta \boldsymbol{\sigma}_{11}^{*}\right|_{\varepsilon=\varepsilon_{\mathrm{ref}}}}{3 G_{\infty} \tilde{g}_{\varepsilon}\left(\varepsilon_{\mathrm{ref}}^{*}\right) \varepsilon_{\mathrm{ref}}^{*}}=\frac{1}{3 G_{\infty}} \frac{\left.\Delta F^{*}\right|_{\varepsilon=\varepsilon_{\mathrm{ref}}}}{A_{0} \tilde{g}_{\varepsilon}\left(\varepsilon_{\mathrm{ref}}^{*}\right) \varepsilon_{\mathrm{ref}}^{*}}
\end{gathered}
$$

In the same manner as for $\tilde{g}_{\varepsilon}(\varepsilon), \tilde{g}_{\omega}(\omega)$ is calculated at a fixed reference dynamic amplitude $\left(\varepsilon_{\text {ref }}=0.5 \%\right)$ and zero static deformation with changing frequencies. The constants $\tilde{g}_{\omega}(\omega)$ are determined by curve fitting using polynomial functions. In this paper, real part of $\tilde{g}_{\omega}(\omega)$ is curve-fitted by a fourth-order polynomial function of $\log$ frequency. And the imaginary part of $\tilde{g}_{\omega}(\omega)$ is curve-fitted by a first-order polynomial function.

Then $c^{*}\left(\mathbf{B}_{0}\right)$ is calculated at fixed reference dynamic amplitude and fixed reference frequency with changing magnitudes of static deformation. The constants $c^{*}\left(B_{0}\right)$ are determined by least-square curve fitting:

$$
c^{*}\left(\mathbf{B}_{0}\right)=\frac{\Delta F^{*}}{\varepsilon_{\mathrm{ref}}^{*}} \frac{\lambda}{A_{0} \tilde{g}_{\omega}\left(\omega_{\mathrm{ref}}\right) \tilde{g}_{\varepsilon}\left(\varepsilon_{\mathrm{ref}}^{*}\right) \mathscr{C}_{\ell}} .
$$

The determined constants are listed in Table 2. Figures 2-4 show the results of the curve fitting.

\subsection{Results and discussion}

The experimental data of the dynamic moduli $E^{\prime}$ and $E^{\prime \prime}$ for zero static deformation are plotted in Figs. 5 and 6 against the strain amplitude for different frequencies. In each figure, $\varepsilon^{*}$ is the dynamic strain and $E^{*}=E^{\prime}+i E^{\prime \prime}$ is the complex Young's modulus. $E^{*}$ is calculated from the measured force and displacement amplitude using the following relation:

$$
E^{*}=\frac{\Delta \sigma_{11}^{*}}{\varepsilon_{x}^{*}}=\left(\frac{\Delta F^{*}}{A_{0} \varepsilon_{x}^{*}}+\frac{F_{0}}{A_{0}}\right) \lambda
$$


Table 2 Identified material constants

\begin{tabular}{ll}
\hline$G_{0}^{\prime}$ & 1.8472 \\
$G_{\infty}^{\prime}$ & 1.0833 \\
$\gamma_{\mathrm{c}}$ & 0.025 \\
$m$ & 0.5 \\
$G_{m}^{\prime \prime}$ & 0.08022 \\
$G_{\infty}^{\prime \prime}$ & 0.01388 \\
$\Delta G_{2}^{\prime \prime}$ & 0.02777 \\
$\gamma_{2}$ & 0.001 \\
$\omega_{\mathrm{ref}}$ & $1 \mathrm{~Hz}$ \\
$a_{1}$ & -0.01509 \\
$a_{2}$ & 0.16868 \\
$a_{3}$ & -0.21523 \\
$a_{4}$ & 0.09712 \\
$b_{1}$ & 0.01056 \\
$z_{\gamma_{1}}$ & -2.92256 \\
$z_{\gamma_{2}}$ & 10.5632 \\
${ }_{\theta}$ & 2.513537 \\
$c_{1}[\mathrm{Mpa}]$ & 0.56 \\
$c_{2}[\mathrm{Mpa}]$ & 0.04 \\
$G_{\infty}[\mathrm{Mpa}]$ & 1.2 \\
$\rho\left[\mathrm{kg} / \mathrm{m}^{3}\right]$ & 1119 \\
\hline
\end{tabular}

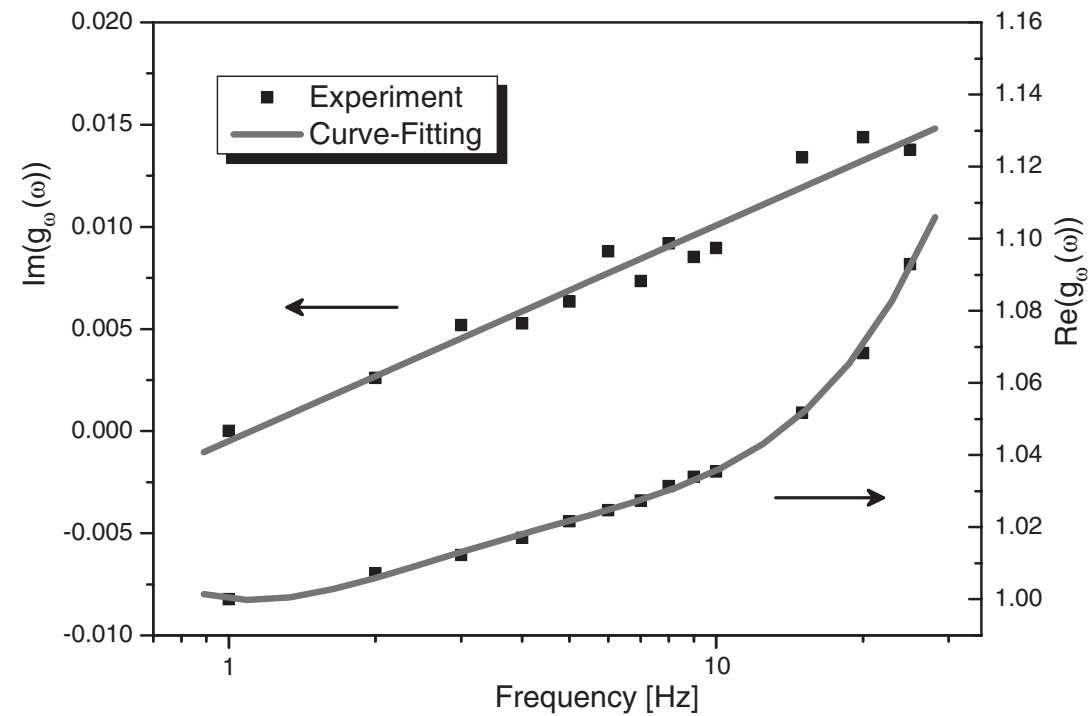

Fig. 2 Frequency influence factor part of relaxation function

where $\Delta F^{*}$ is the dynamic force amplitude, $F_{0}$ the static force, and $A_{0}$ the cross-section area of a specimen in an undeformed state.

We observe a pronounced decrease in the storage modulus and a sigmoidal behavior of the loss modulus with increasing strain amplitudes [10-13]. Since the storage modulus is a measure of the stiffness of the material, it is proportional to the number of intact physical bonds that can transfer forces on the microscopic scale. Its monotonic decline is caused by a decrease in the number of intact bonds with increasing amplitudes. As we know, the loss modulus is proportional to the breakage rate of the bonds. Since the rate of breakage is 


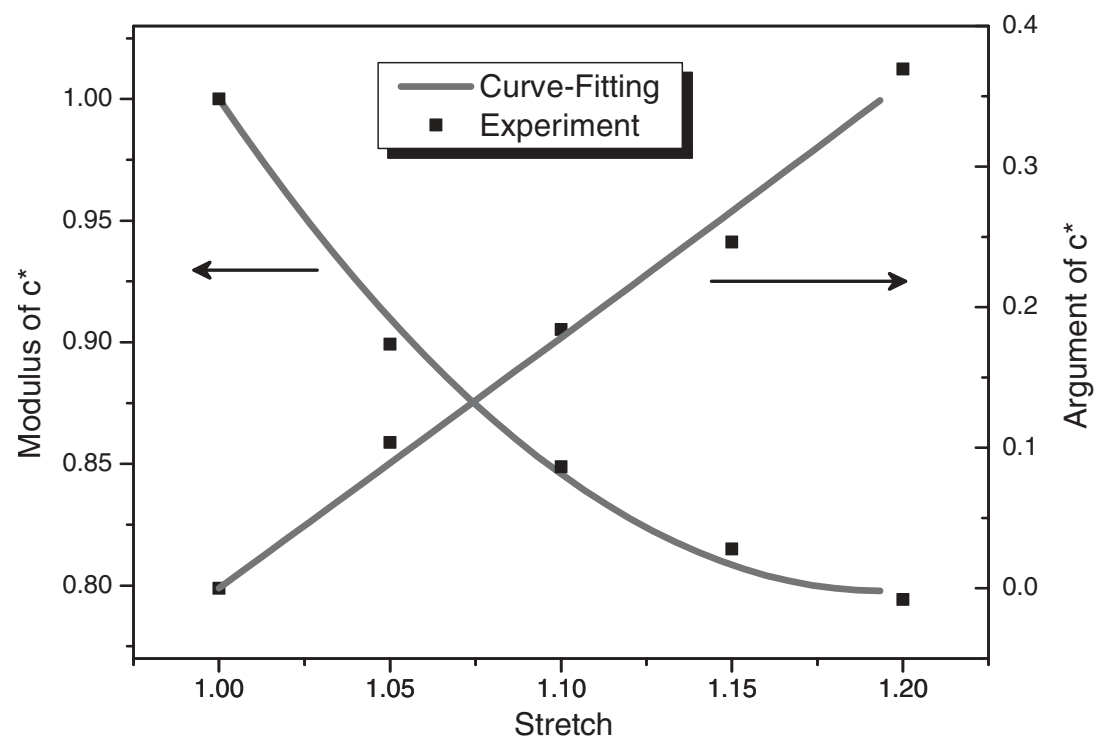

Fig. 3 Static deformation influence factor part of relaxation function

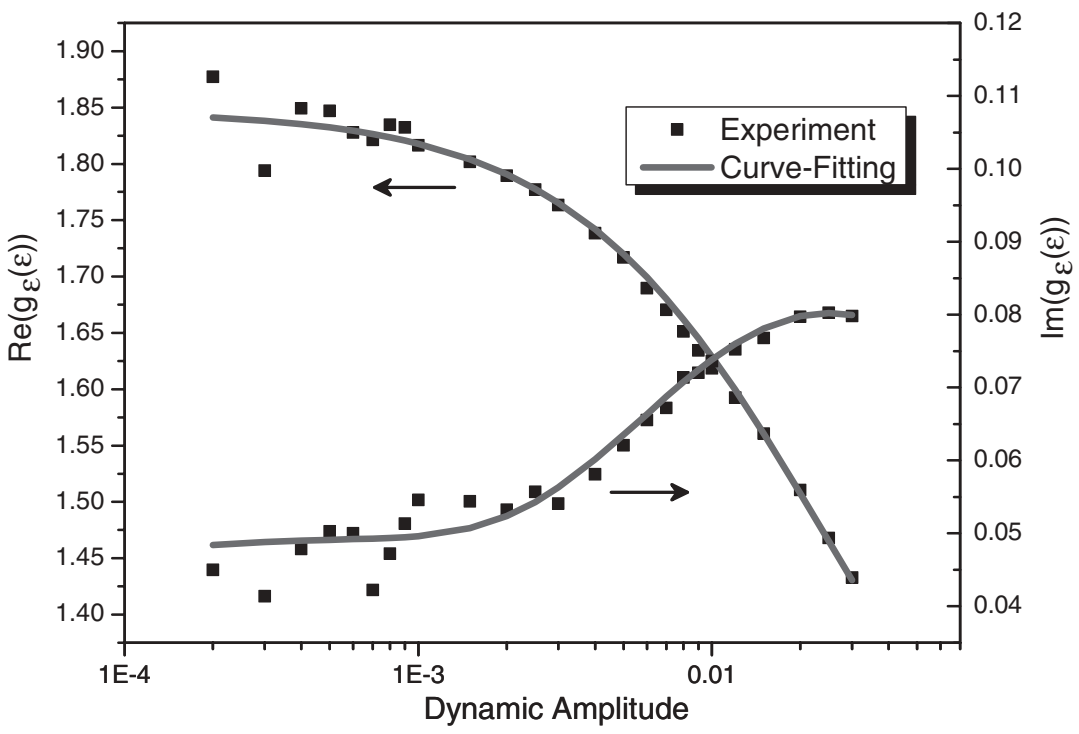

Fig. 4 Dynamic deformation influence factor part of relaxation function

proportional to the number of intact bonds and increases with increasing amplitudes, we observe a sigmoidal behavior: on the ascending side of the curves, the energy loss increases with the amplitude because there are enough intact bonds; on the descending side of the curves the energy loss decreases with the amplitude because the number of intact bonds has considerably decreased. As we observe in the plots, the frequency dependency is monotonic in both cases. That is, increasing frequencies lead to an increase in stiffness and an increase in energy loss per cycle. The increase in dynamic modulus at higher frequencies reflects the reduced molecular motions of the elastomer chains [20].

Comparing the experimental data with those of the proposed model shown in Figs. 5 and 6, it can be seen that the model produces results comparable to the experimental data. And the parameters of $\tilde{g}_{\varepsilon}(\varepsilon)$ used in Figs. 5 and 6 are determined by the values at $1 \mathrm{~Hz}$. Figures 5 and 6 show that the value of $\tilde{g}_{\varepsilon}(\varepsilon)$ determined at $1 \mathrm{~Hz}$ is also effective at other frequencies. The responses at different frequencies could be equally well predicted. This confirms the author's assumption that $\tilde{g}_{\varepsilon}(\varepsilon)$ and $\tilde{g}_{\omega}(\omega)$ are separable. 


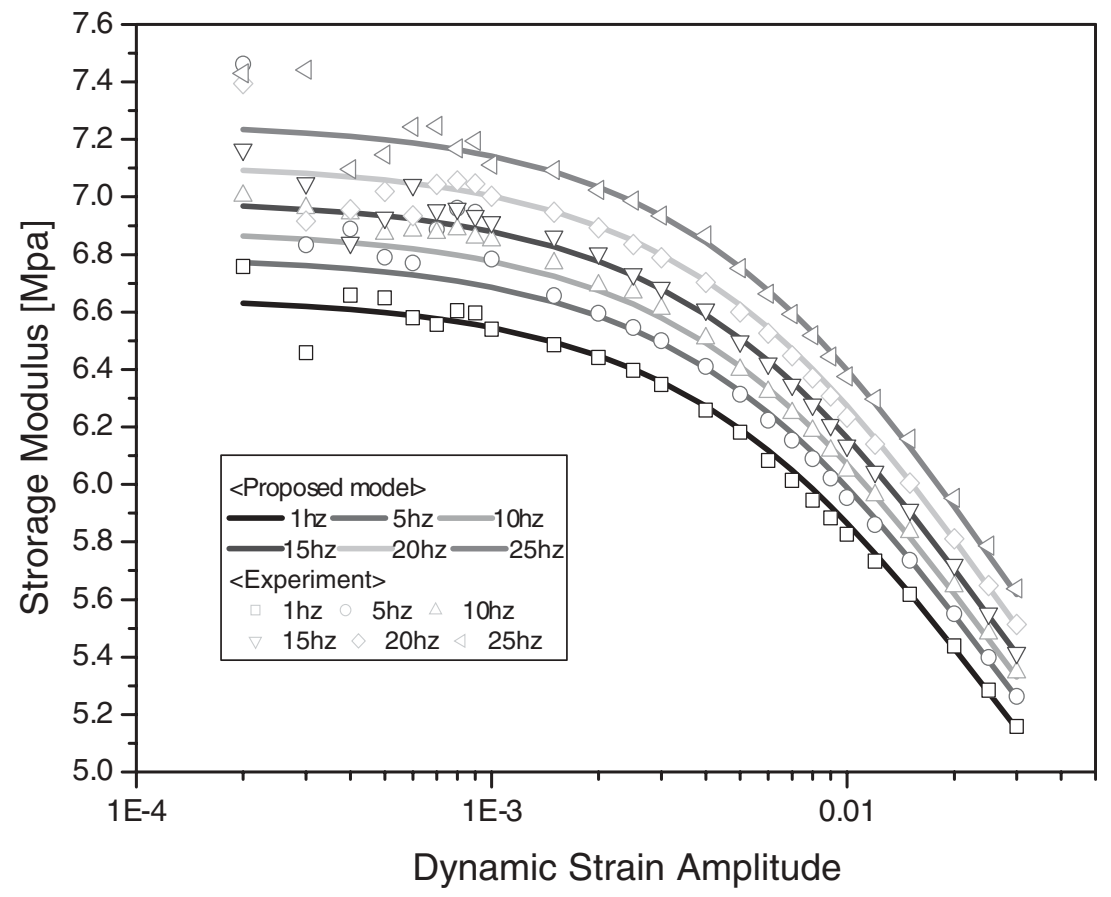

Fig. 5 Storage modulus belonging to no static deformation against dynamic strain amplitude for different frequencies in uniaxial tension test

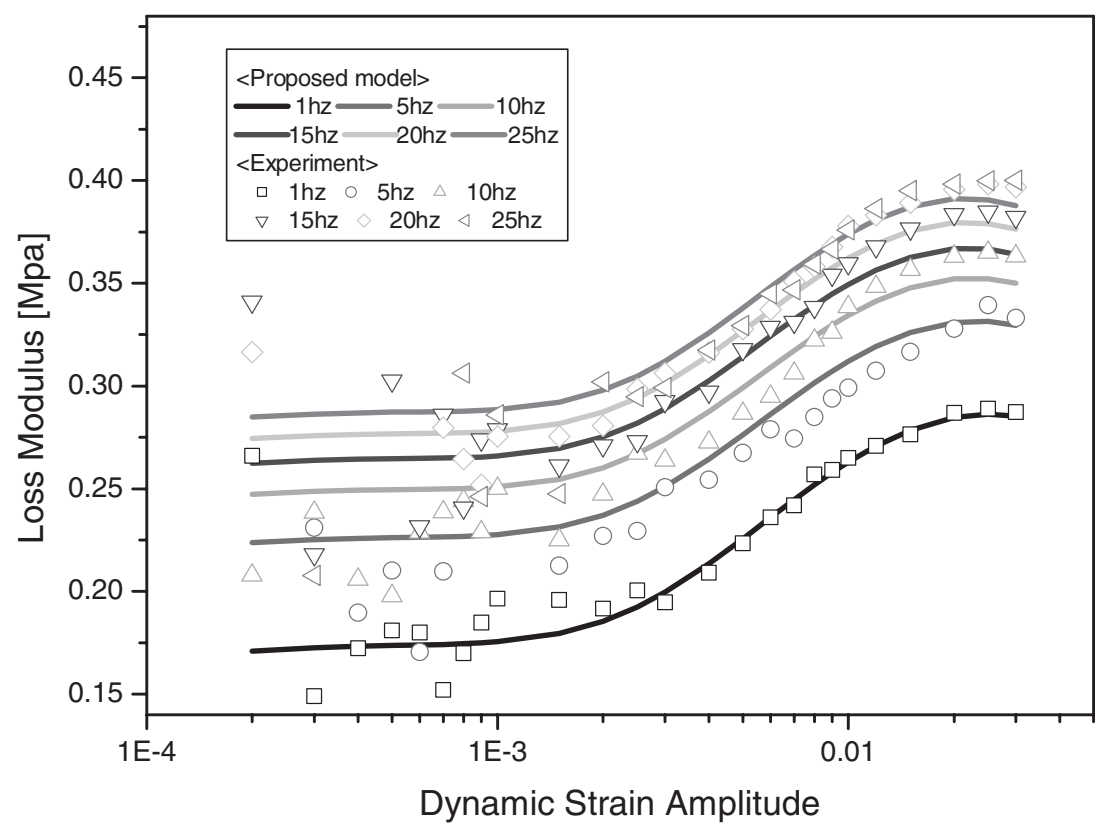

Fig. 6 Loss modulus belonging to no static deformation against dynamic strain amplitude for different frequencies in uniaxial tension test

In Figs. 7 and 8, the experimental data of the dynamic moduli $E^{\prime}$ and $E^{\prime \prime}$ for $0.5 \%$ dynamic strain are plotted against the static deformation for different frequencies. In these figures, we observe the initial decrease in storage and loss moduli and the subsequent increase at higher strain [6,7]. The initial decrease in storage and loss moduli is due to the disruption of the filler network, and the subsequent increase at higher strain is attributed to the limited extensibility of the elastomer chains [6]. For the explanation of this effect, a static 


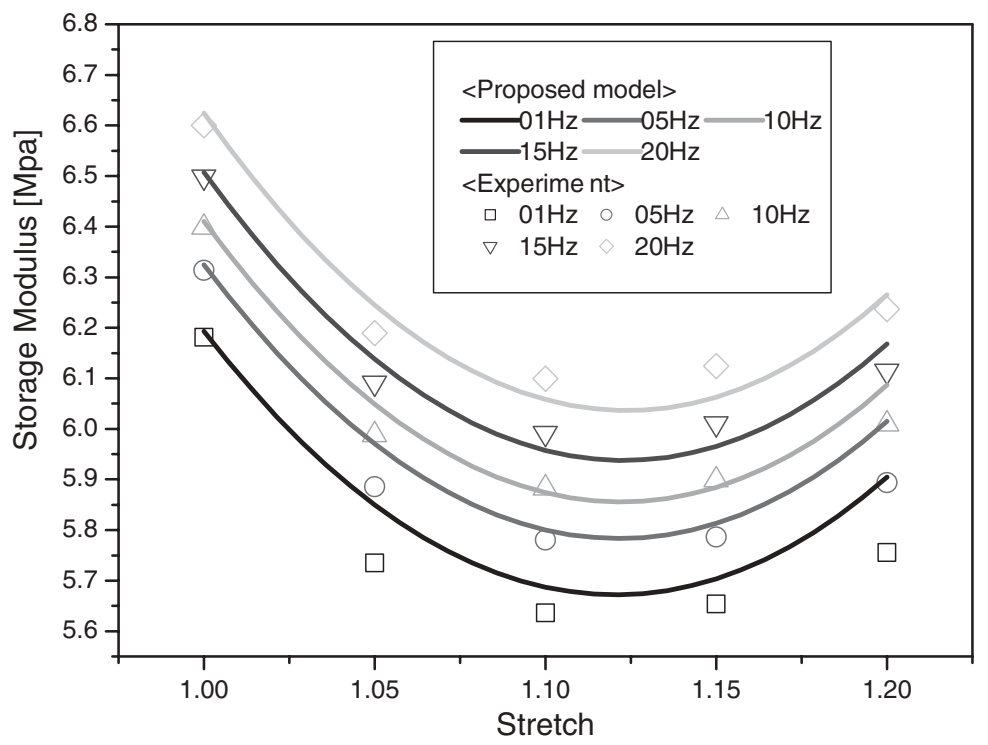

Fig. 7 Storage modulus belonging to $0.5 \%$ dynamic strain against static deformation for different frequencies in uniaxial tension test

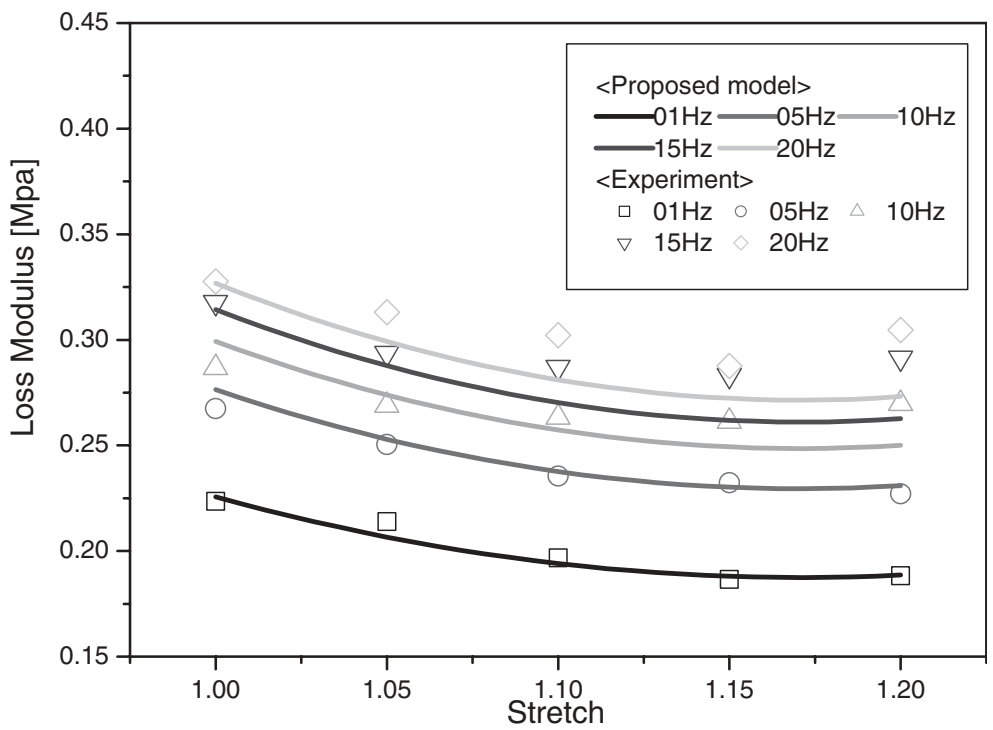

Fig. 8 Loss modulus belonging to $0.5 \%$ dynamic strain against static deformation for different frequencies in uniaxial tension test

deformation influence factor $c^{*}$ was introduced in the $\mathrm{K}-\mathrm{Y}$ model [7]. As is the case in the $\mathrm{K}-\mathrm{Y}$ model, this constitutive equation with a static deformation influence factor shows good agreement with test results. The value of $c^{*}$ used in Figs. 7 and 8 is determined by the values at $10 \mathrm{~Hz}$ and $0.5 \%$ dynamic strain. Figures 7 and 8 show that the value of $c^{*}$ determined at $10 \mathrm{~Hz}$ is also valid at other frequencies. With the static deformation influence factor determined at one frequency, the responses at different frequencies could be equally well predicted. This confirms the author's assumption that $c^{*}$ is only a function of static deformation.

Finally, the relation between SDIF and DAIF will be discussed. In Fig. 9, the calculated storage modulus is compared with the experimental results. The experimental data of the storage modulus at $10 \mathrm{~Hz}$ are plotted against the static deformation for different dynamic strains. In the case of prestrain and dynamic amplitude, however, the correlation between two factors cannot be entirely ignored. The proposed model also shows this. The same statement holds for the representation of the loss modulus shown in Fig. 10. The correlation between 


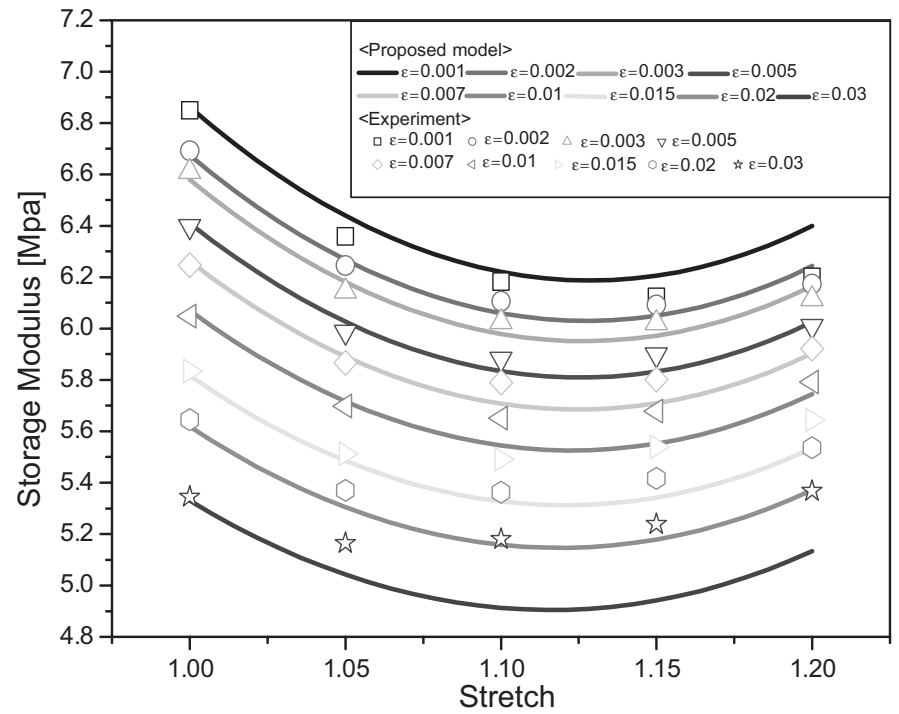

Fig. 9 Storage modulus belonging to $10 \mathrm{~Hz}$ frequency against static deformation for different dynamic strains in uniaxial tension test

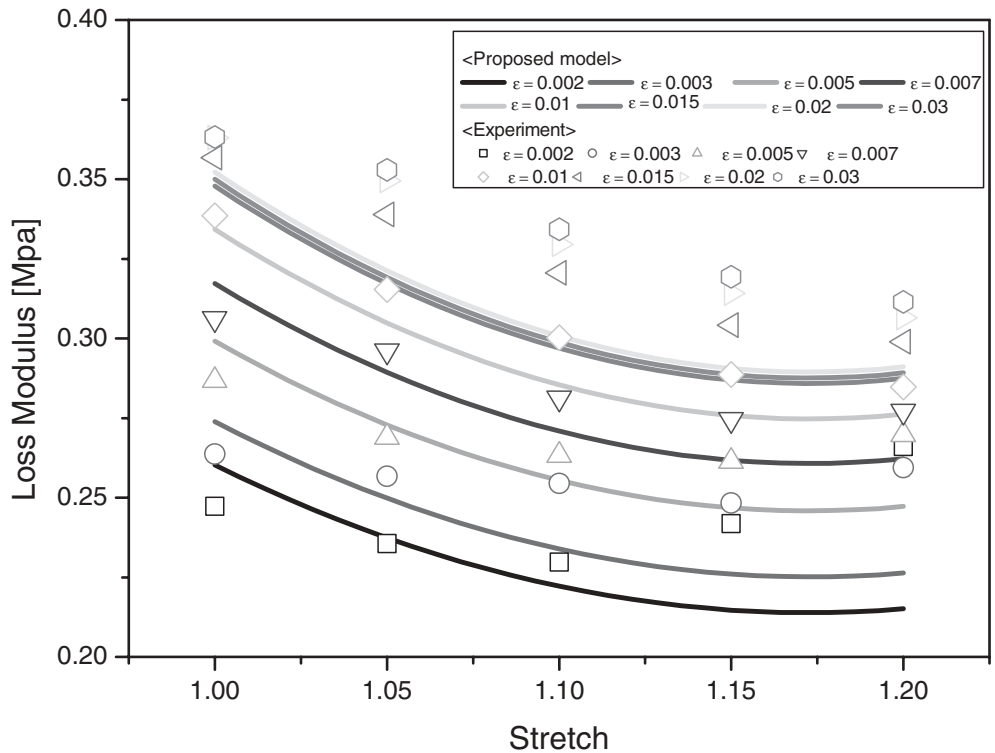

Fig. 10 Loss modulus belonging to $10 \mathrm{~Hz}$ frequency against static deformation for different dynamic strains in uniaxial tension test

prestrain and dynamic amplitude must be carefully studied. A study of the coupling of these two factors remains a future endeavour.

\section{Conclusion}

An effective and practical viscoelastic constitutive equation of filled rubber considering the Payne effect as well as the effects of large static prestrain and frequency is proposed. The proposed constitutive model is based on the linearization of Simo's finite viscoelastic model and reference configuration transformation. Influence factors are introduced in this model in order to consider the influence of static predeformation, the dynamic-strain-dependent properties, and frequency-dependent properties. For simplicity, the separation of 
variables - frequency, predeformation, and dynamic strain —is assumed. The Kraus model is used for describing the dynamic-strain-dependent properties.

Dynamic tension tests are executed to obtain the model parameters and also for the verification of the model. The suggested constitutive equation shows good agreement with test values. Separability among the three factors is assumed in the model. However, in the case of prestrain and dynamic amplitude, the correlation between the two factors cannot be entirely ignored. A study of this coupling effect remains a future endeavour.

Acknowledgements This work was supported by Grant No. R01-2001-000-00393 from the Basic Research Program of the Korea Science\& Engineering Foundation.

\section{References}

1. Sulivan, J.I., Morman, K.N., Pett, R.A.: A non-linear viscoelastic characterization of a natural rubber gum vulcanizate. Rubber Chem Technol 53, 805-822 (1980)

2. Morman, K.N., Jr Nagtegaal, J.C.: Finite element analysis of sinusoidal small-amplitude vibrations in deformed viscoelastic solids. Part I: Theoretical development. Int J Numer Methods Eng 19, 1079-1103 (1983)

3. Zdunek, A.B.: Theory and computation of the steady state harmonic response of viscoelastic rubber parts. Comput Meth Appl Mech Eng 105, 63-92 (1993)

4. Zdunek, A.B.: Determination of material response functions for prestrained rubbers. Rheol Acta 31, 575-591 (1992)

5. Hibbit, Karlsson and Sorenson Inc. ABAQUS theory manual, version 5.7 (1997)

6. Voet, A., Morawski, J.C.: Dynamic mechanical and electrical properties of vulcanizates at elongations up to sample rupture. Rubber Chem Technol 47, 765-777 (1974)

7. Kim, B.-K., Youn, S.-K.: A viscoelastic constitutive model of rubber under small oscillatory load superimposed on large static deformation. Arch Appl Mech Ingenieur Archiv 12(71), 748-763 (2001)

8. Simo, J.C.: A fully three-dimensional finite-strain viscoelastic damage model: formulation and computational aspects. Comput Meth Appl Mech Eng 60, 153-173 (1987)

9. Kim, B.-K., Youn, S.-K., Lee, W.-S.: A constitutive model and fea of rubber under small oscillatory load superimposed on large static deformation. Arch Appl Mech Ingenieur Archiv 73, 781-798 (2004)

10. Payne, A.R.: The dynamic properties of carbon black-loaded natural rubber vulcanizates. Part I. J Appl Polym Sci 6(19), 57-63 (1962)

11. Payne, A.R.: The dynamic properties of carbon black loaded natural rubber vulcanizates. Part II@. J Appl Polym Sci 6(21), 368-372 (1962)

12. Payne, A.R.: Strainwork dependence of filler-loaded vulcanizates. J Appl Polym Sci 8(6), 2661-2686 (1965)

13. Payne, A.R.: A note on the conductivity and modulus of carbon black-loaded rubbers. J Appl Polym Sci 9(3), 1073-1082 (1965)

14. Kraus, G.J.: Applied polymer symposium. J Appl Polym Sci 39, 75 (1984)

15. Ulmer, J.D.: Strain dependence of dynamic mechanical properties of carbon black-filled rubber compounds. Rubber Chem Technol 69, 15-47 (1996)

16. Christensen, R.M.: Theory of viscoelasticity. Academic, New York (1982)

17. Truesdell, C., Noll, W.: The non-linear field theories of mechanics. In: Flügge S (ed) Encyclopedia of physics. Springer, Berlin Heidelberg New York (1965)

18. Jung, G.D., Youn, S.K., Kim, B.K.: A three dimensional nonlinear viscoelastic constitutive model of solid propellant. Int J Solids Struct 37, 4715-4732 (2000)

19. Brown, R.P.: Physical testing of rubber, 3rd edn. Chapman \& Hall, London (1996)

20. Ferry, J.D.: Viscoelastic properties of polymers. Wiley, New York (1980)

21. Washizu, K.: Variational methods in elasticity plasticity, 3rd edn. Pergamon, Oxford (1982) 